\title{
Hábitos de Nidificação de Polistes canadensis (Linnaeus) (Hymenoptera: Vespidae) em Área Urbana
}

\author{
Francisco Virgínio', Tatiane Tagliatti Maciel² \& Bruno Corrêa Barbosa ${ }^{\bowtie}$
}

1. Universidade Federal do Rio Grande do Norte, Natal, Rio Grande do Norte, Brasil, e-mail: f.vs1987@hotmail.com. 2. Laboratório de Ecologia Comportamental e Bioacústica (LABEC ) - Universidade Federal de Juiz de Fora - MG - Brasil, e-mail: tatitagliatti@hotmail.com, barbosa.bc@outlook.com (Autor para correspondência ${ }^{\bowtie}$ ).

\section{EntomoBrasilis 9 (2): 81-83 (2016)}

Resumo. Visando compreender a ecologia de nidificação de Polistes canadensis (Linnaeus) em áreas urbanas, foram realizadas buscas ativas por colônias no Campus da Universidade Federal do Rio Grande do Norte. Foram encontradas 442 colônias em sete materiais como substrato para nidificação, sendo $98 \%$ em edificações humanas. Concreto e metal foram os substratos mais utilizados para nidificação das vespas, podendo ser justificado pela maior presença desses materiais no ambiente e ainda, pela retenção de calor que eles apresentam fornecendo condição favorável para o desenvolvimento das colônias.

Palavras-Chaves: Arquitetura de ninhos; colônias; ecologia de paisagem; insetos sociais.

\section{Nesting habits of Polistes canadensis (Linnaeus) (Hymenoptera: Vespidae) in Urban Areas}

Abstract. Aiming to understand nesting ecology of Polistes canadensis (Linnaeus) in urban areas, were carried out active searches for colonies on the campus of the Universidade Federal do Rio Grande do Norte. We found 442 colonies in seven materials as substrate for nesting, $98 \%$ in human buildings. Concrete and metal were the most commonly used substrates by the wasps nidifying and this fact can be justified by the greater presence of these in the environment and also the heat retention they present providing favorable condition for the development of the colonies.

Keywords: Colonies; landscape ecology; nest architecture; social insects.

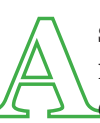
s vespas possuem uma importância ecológica e econômica relevante para o ecossistema urbano e natural, pois atuam como polinizadoras, bioindicadoras e como controladoras naturais de insetos herbívoros em agroecossistemas (РRеzoto et al. 2008; Souza et al. 2010; Clemente et al. 2012; Barbosa et al. 2014).

As vespas do gênero Polistes (Hymenoptera: Vespidae) apresentam fundação de colônias do tipo independente, na qual a construção do ninho é feita por apenas uma fêmea inseminada ou por um pequeno grupo de fêmeas fecundadas que ovipositam e alimentam as larvas. De um modo geral, após a emersão de novos indivíduos, a fêmea dominante passa a realizar apenas a atividade reprodutora, e as outras vespas passam a atuar como operárias, realizando as atividades de forrageio, manutenção e ampliação da colônia (NASCIMENTO et al. 2008; CARPENTER \& MARQues 2001). Seus ninhos são constituídos geralmente por apenas um favo, sem envelope protetor, apresentando um pedúnculo curto preso ao substrato, revestido com substâncias repelentes a formigas (WENZEL 1998; CARPENTER \& MARQUES 2001).

Em áreas urbanas, vários estudos demonstram que vespas do gênero Polistes utilizam principalmente substratos artificiais para nidificação, ainda que substratos naturais estejam presentes no ambiente (Butignol 1992; Giannotti \& Mansur 1993; Sinzato \& Prezoto 2000; Barbosa 2015). Na literatura são citados diferentes substratos feitos pelo homem que são utilizados por vespas do gênero Polistes para a fundação de suas colônias, como madeira, metal, concreto, vidro e telhas de amianto (FERREIRA \& Sinzato 2007; Torres et al. 2009; Oliveira et al. 2010; Barbosa 2015). Segundo Fowler (1983) Polistes spp. ocorrem com maior frequência em ambiente urbano quando comparado ao ambiente natural, porque as edificações humanas proporcionam substratos mais adequados para fixação de colônias. Entretanto, ainda há pouco conhecimento a respeito da utilização dos substratos artificiais pelas vespas sociais e a relação das propriedades de cada material com as colônias.

Sendo assim o presente estudo tem como objetivo registrar os substratos utilizados para nidificação da Polistes canadensis (Linnaeus), a fim de compreender a ecologia do grupo em áreas urbanas assim como avaliar a existência de um padrão de nidificação com tendência a algum substrato.

\section{MATERIAL E MÉTODOS}

Área de Estudo. O estudo foi desenvolvido no Campus Central da Universidade Federal do Rio Grande do Norte ( $5{ }^{\circ} 50$ '24"S $35^{\circ} 11^{\prime} 56^{\prime \prime W)}$, que ocupa uma área de 123 ha e está localizado na porção sul da cidade de Natal, no estado do Rio Grande do Norte circundado por vegetação nativa e exótica, caracterizando uma área verde urbana (MACIEL \& BARBOSA 2015). A região apresenta clima quente úmido, ocorrendo o período chuvoso entre os meses de abril a setembro e o período seco entre os meses de outubro a março (CARVALHO 2005).

Coleta de dados. Foi utilizado o método de busca ativa por colônias, durante o mês de maio de 2015 no intervalo entre 8:0o e 12:0o h durante duas semanas. O método consistiu em procurar colônias nos jardins e em construções humanas no Campus da Universidade Federal do Rio Grande do Norte. As colônias encontradas foram fotografadas e tiveram a altura estimada; o material usado como substrato e a área de nidificação foram registrados. Também foram coletados alguns indivíduos com 
rede entomológica, que foram armazenados em recipiente com álcool 70\% devidamente etiquetados, para posterior confirmação da identificação.

Para gerar o grafo entre as colônias de $P$. canadensis e seus substratos de nidificação no Campus da Universidade Federal do Rio Grande do Norte, foram analisadas todas as colônias observadas e sete tipos de substratos, considerando a frequência das nidificações sobre cada substrato, utilizando o software $\mathrm{R}$ CoreTeam (freeware).

\section{RESULTADOS}

Foram registradas 442 colônias de $P$. canadensis em sete materiais como substrato para nidificação, sendo $98 \%(n=433)$ do total de colônias, fundadas em edificações humanas. Apenas nove colônias foram registradas em vegetação, dessas, cinco foram encontradas em Clitoria fairchildiana R. A. Howard. (Fabaceae) e quatro em galhos de Licania tomentosa (Benth.) Fritsch (Chrysobalanaceae).

Os substratos artificiais utilizados para nidificação de $P$. canadensis no Campus da Universidade Federal do Rio Grande do Norte, observados nesse estudo foram: concreto $(\mathrm{n}=314)$; metal ( $\mathrm{n}=78)$; telha de amianto $(\mathrm{n}=25)$; plástico $(\mathrm{n}=13)$; madeira $(n=2)$ e telha de barro $(n=1)$ (Figura 1$)$.

Apenas três colônias foram registradas abaixo de dois metros de altura, todas as demais foram fundadas a pelo menos $2 \mathrm{~m}$ do nível do solo, sendo registradas algumas colônias a cerca de $7 \mathrm{~m}$ de altura.

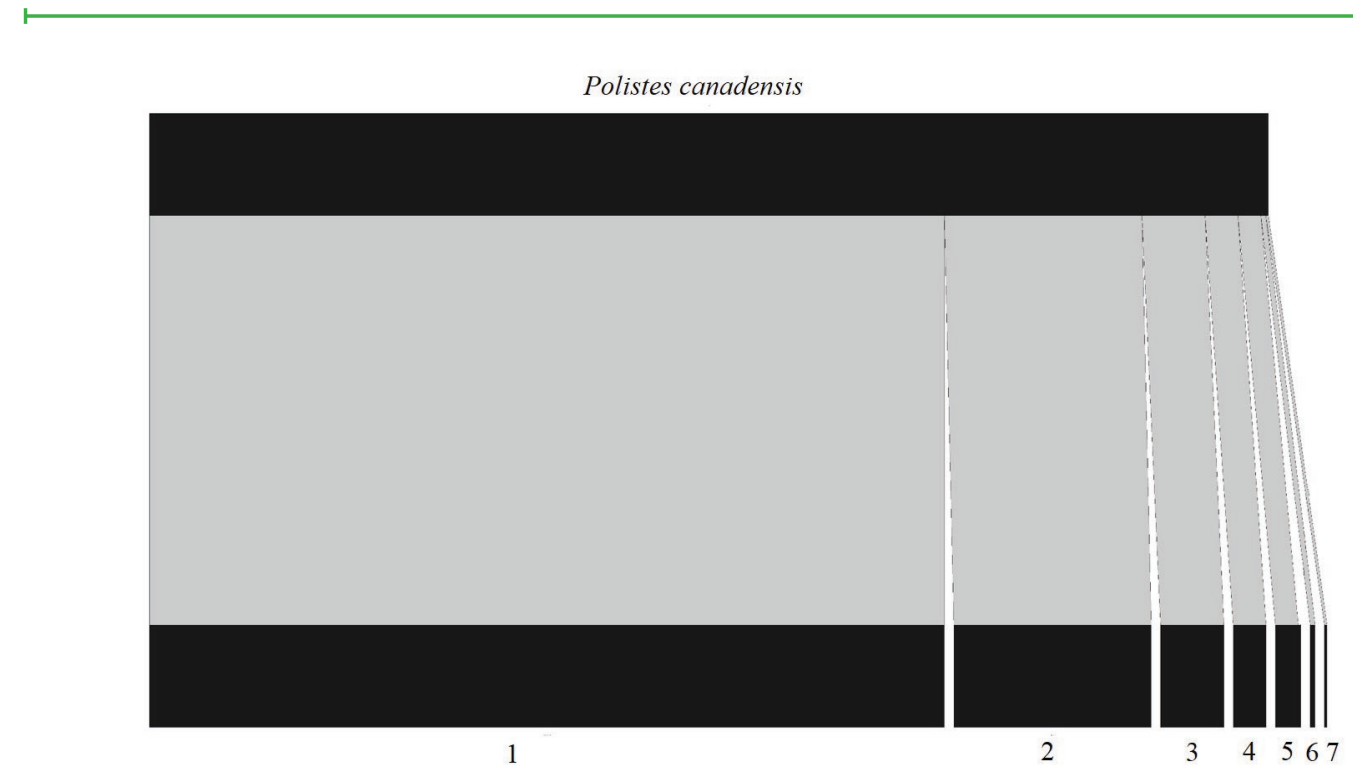

Figura 1. Grafo relacionando a nidificação entre Polistes canadensis (Hymenoptara: Vespidae) e substratos artificiais utilizados no Campus da Universidade Federal do Rio Grande do Norte. Legenda: 1 - Concreto; 2 - Metal; 3 - Telha de Amianto; 4 - Plástico; 5 - Vegetação; 6 - Madeira; 7 - Telha de Barro.

\section{DISCUSSÃO}

As plantas utilizadas como substrato de nidificação de $P$. canadensis apresentam condições favoráveis para nidificação de vespas sociais (WENZEL 1998; BARBOSA 2015) como porte arbustivo, que oferece defesa contra ventos e chuvas e folhas largas, que possuem maior superfície de fixação para as colônias. Além disso, estavam localizadas ao lado das edificações com grande presença de colônias da mesma espécie, sugerindo que as colônias construídas na vegetação se deram provavelmente ao grande número de colônias de $P$. canadensis nas edificações, uma vez que vespas menos agressivas perdem espaço para as mais agressivas.

A alta taxa de nidificação em concreto pode ser justificada pelo fato de ser o substrato artificial mais abundante no local, indicando uma adaptação das populações de $P$. canadensis do Campus a ambientes urbanos utilizando os materiais mais disponíveis. Outras espécies de vespas sociais de fundação independente também mostram uma tendência de nidificação em edificações humanas quando comparada à vegetação em áreas urbanas, como registrado por Lima et al. (2000), ALVARENGA et al. 2010 e BARBosa (2015). É possível inferir ainda que a adaptação dos hábitos de nidificação das vespas sociais em áreas urbanas aumenta as chances de sobrevivência desses insetos, pois essas proporcionam uma maior protecão das colônias contra intempéries climáticas, menor pressão de predação por inimigos naturais e uma menor pressão de competição entre as espécies, devido à baixa diversidade que ocorre nesses ambientes (PrEzoto et al. 2007).
Os substratos mais utilizados (concreto e metal) podem também estar relacionados à ocorrência de um fenômeno chamado de "ilha de calor" (UcHÔA 2011), onde acontece um acúmulo de calor nesses tipos de material proporcionando condições adequadas para o desenvolvimento das colônias, fato já registrado para formigas por FERNANDES et al. (2014). A baixa taxa de nidificação nos demais materiais (telha de amianto, madeira, plástico e telha de barro) pode ser justificada pela menor disponibilidade deles no ambiente.

Todas as colônias encontradas nas edificações humanas estavam abrigadas, protegidas da incidência direta da luz solar e da chuva. A maioria das colônias foi localizada no lado leste das edificações, área que recebe nas primeiras horas do dia intensa radiação solar, o que pode indicar o uso do calor ao amanhecer para acelerar o metabolismo, iniciando as atividades no período mais cedo do dia, sendo essa relação da influência da temperatura na atividade das vespas bem documentada na literatura (CASTRO et al. 2011; ELISEI et al. 2013; YeISON et al. 2013; DETONi et al. 2015).

Quanto às alturas das colônias registradas pelo presente estudo, é possível inferir grande adaptação da espécie ao ambiente urbano, tendo em vista que ninhos acima de $2 \mathrm{~m}$ de altura sofrem menos injúrias de transeuntes.

Os resultados corroboram estudos que apontam a tendência de nidificação de Polistes spp. em substratos artificiais demonstrando sua alta sinantropia. Com isso, e tendo em vista o papel ecológico que as vespas sociais desempenham, fica clara a importância de 
mais estudos sobre a dinâmica das populações desses insetos em ambientes urbanos.

\section{REFERÊNCIAS}

Alvarenga, R.B., M.M. Castro, E.H. Santos-Prezoto \& F. Prezoto, 2010. Nesting of social wasps (Hymenoptera, Vespidae) in urban gardens in southeastern Brazil. Sociobiology, 55: 445452.

Barbosa, B.C., 2015. Vespas sociais (Vespidae: Polistinae) em fragmento urbano: riqueza, estratificação e redes de interação. Dissertação (Mestrado em Ciências Biológicas: Zoologia) Universidade Federal de Juiz de Fora, Brasil. 60 f.

Barbosa, B.C., M.F. Paschoalini \& F. Prezoto, 2014. Temporal activity patterns and foraging behavior by social wasps (Hymenoptera, Polistinae) on fruits of Mangiferaindica L. (Anacardiaceae). Sociobiology, 61: 239-242. DOI: http://dx.doi.org/10.13102/sociobiology.v61i2.239-242.

Butignol, C.K., 1992. Observações sobre a biologia da vespa predadora Polistes versicolor (Olivier, 1791) (Hymenoptera: Vespidae) em Florianópolis/SC. Anais da Sociedade Entomológica do Brasil, 21: 113-123.

Carpenter, J.M. \& O.M. Marques, 2001. Contribuição ao estudo dos vespídeos do Brasil (Insecta, Hymenoptera, Vespoidea, Vespidae). Publicações Digitais, 2: 1-147.

Carvalho, S.O., 2005. Análise bioclimática como ferramenta para implementação do Plano Diretor do Campus Central da UFRN. Dissertação (Mestrado em Arquitetura e Urbanismo) - Universidade Federal do Rio Grande do Norte, Brasil. $171 \mathrm{f}$.

Castro, M.M., D.L. Guimarães \& F. Prezoto, 2011. Influence of Environmental Factors on the Foraging Activity of Mischocyttarus cassununga (Hymenoptera, Vespidae). Sociobiology, 58: 133-141.

Clemente, M.A., D. Lange, K. Del-Claro, F. Prezoto, N.R. Campos \& B.C. Barbosa, 2012. Flower-visiting social wasps and plants interaction: Network pattern and environmental complexity. Psyche: A Journal of Entomology, 2012: 1-10. DOI: http://dx.doi.org/10.1155/2012/478431.

Detoni, M., M.C. Mattos, M.M. Castro, B.C. Barbosa \& F. Prezoto, 2015. Activity schedule and foraging in Protopolybia sedula (de Sausurre, 1854) (Hymenoptera, Vespidae). Revista Colombiana de Entomologia, 41: 245-248.

Elisei, T., J. Nunes, C. Ribeiro JR., A. Fernandes JR. \& F. Prezoto, 2013. What is the Ideal Weather for Social Wasp Polistes versicolor (Olivier) go to Forage? EntomoBrasilis, 3: 214-216. DOI: http://dx.doi.org/10.12741/ebrasilis.v6i3.342.

Fernandes, E.F., M.M. Castro, B.C. Barbosa \& F. Prezoto, 2014. Variation in nesting behavior of the arboreal ant Camponotus sericeiventris (Hymenoptera: Formicidae). Florida Entomologist, 97: 1237-1239. DOI: http://dx.doi. org/10.1653/024.097.0332.

Ferreira, E.L. \& D.M.S. Sinzato, 2007. Ocorrência e sítio de nidificação de vespas sociais Polistes sp. (Hymenoptera, Vespidae) no Parque Aquático Águas Quentes, Barra do Piraí/ RJ. Anais do VIII Congresso de Ecologia do Brasil, CaxambuMG, Brasil, p 1-2.

Fowler, H.G., 1983. Human effects on nest survivorship of urban synanthropic wasps. Urban Ecology, 7: 137-143. DOI: http://dx.doi.org/10.1016/0304-4009(83)90032-3.
Giannotti, E. \& C.B. Mansur, 1993. Dispersion and foundation of new colonies in Polistes versicolor (Hymenoptera, Vespidae). Anais da Sociedade Entomológica do Brasil, 22: 307-316.

Lima, M.A.P., JR. Lima \& F. Prezoto, 2000. Levantamento dos gêneros de vespas sociais (Hymenoptera, Vespidae), flutuação das colônias e hábitos de nidificação no campus da UFJF, Juiz de Fora, MG. Revista Brasileira de Zoociências, 2: 69-80.

Maciel T.T. \& B.C. Barbosa, 2015. Áreas verdes urbanas: história, conceitos e importância ecológica. CES Revista, 29: 30-42.

Nascimento, F.S., I.C. Tannure-Nascimento \& S. Mateus, 2008. Vespas sociais neotropicais: padrões comportamentais, regulação social e arquitetura de ninho, p. 47-67. In: Vilela, E.F., I.A. Santos, J.H. Schoereder, J.E. Serrão, L.A.O. Campos \& J. Lino-Neto (Eds.). Insetos Sociais: da biologia à aplicação. Viçosa, Ed. UFV, $442 \mathrm{p}$

Oliveira, S.A., M.M. Castro \& F. Prezoto, 2010. Foundation pattern, productivity and colony success of the paper wasp, Polistes versicolor. Journal of Insect Science, 10:125. DOI: http://dx.doi.org/10.1673/031.010.12501.

Prezoto, F., C. Ribeiro-Júnior, S.A.O. Cortes \& T. Elisei, 2007. Manejo de vespas e marimbondos em ambiente urbano, $\mathrm{p}$. 123-126. In: Pinto, A.S., M.M. Rossi \& E. Salmeron (Eds.). Manejo de Pragas Urbanas. vol 1. Piracicaba, CP2.

Prezoto, F., S.A.O. Cortes \& A.C. Melo, 2008. Vespas: de vilãs a parceiras. Ciência Hoje, 48: 70-73.

RCore Team, 2013. R: A Language and Environment for Statistical Computing. R Foundation for Statistical Computing, Vienna, Austria. ISBN 3-900051-07-0. http://www.R-project.org/.

Sinzato, D.M.S. \& F. Prezoto, 2000. Aspectos comportamentais de fêmeas dominantes e subordinadas de Polistes versicolor Olivier, 1791 (Hymenoptera: Vespidae) em colônias na fase de fundação. Revista de Etologia, 2: 121-127.

Souza, M.M., J. Louzada, J.E. Serrão \& J.C. Zanuncio, 2010. Social wasps (Hymenoptera:Vespidae) as indicators of conservation degree of riparian forests in Southeast Brazil. Sociobiology, 56: 387-396.

Torres, V.O., T.S. Montagna, G. Bortoluzzi \& W.F. AntonialliJunior, 2009. Aspectos bionômicos da vespa social Neotropical Polistes canadensis canadensis (Linnaeus) (Hymenoptera, Vespidae). Revista Brasileira de Entomologia, 53: 134-138. DOI: http://dx.doi.org/10.1590/s008556262009000100028.

Uchôa, P.W.S., 2011. Estudo de variações termo higrométricas em cidade equatorial devido ao processo de urbanização: $\mathrm{O}$ caso de Belém-PA. Tese de Doutorado, Escola de Engenharia de São Carlos. Universidade de São Paulo. 77 f.

Wenzel, J.W., 1998. A generic key to the nests of hornets, yellow jackets, and paper wasps worldwide (Vespidae: Vespinae, Polistinae). American Museum Novitates, 3224: 1-39.

Yeison, L.G., J.D. Hernandez \& P. Caraballo, 2013. Actividad de forrajeo de la avispa social Polybia emaciata (Hymenoptera: Vespidae: Polistinae). Revista Colombiana de Entomologia, 39: 250-255.

Recebido em: 21.i.2016

Aceito em: 27.iv.2016

Como citar este artigo:

Virgínio, F., T.T. Maciel \& B.C. Barbosa, 2016. Hábitos de Nidificação de Polistes canadensis (Linnaeus) (Hymenoptera: Vespidae) em Área Urbana. EntomoBrasilis, 9 (2): 81-83.

Acessível em: doi:10.12741/ebrasilis.vgi2.586
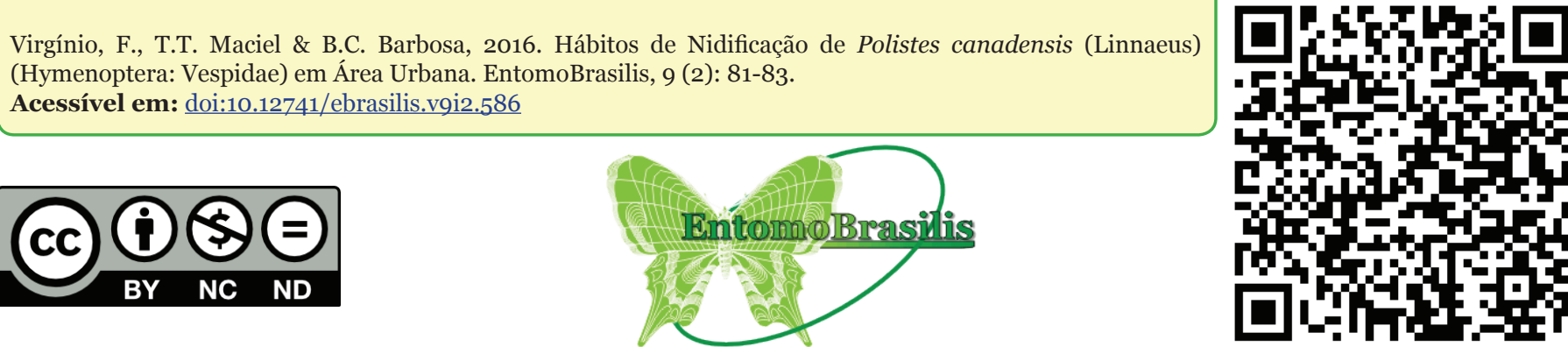